\title{
Outer Synchronization of a Modified Quorum-Sensing Network via Adaptive Control
}

\author{
Jianbao Zhang $\mathbb{D}^{1,2,3}$ Wenyin Zhang, ${ }^{1,2}$ Denghua Zhang, \\ Chengdong Yang $(1),{ }^{1,2,3}$ Kongwei Zhu, ${ }^{1,2}$ and Jianlong Qiu ${ }^{2,5}$ \\ ${ }^{1}$ School of Information Science and Engineering, Linyi University, Linyi 276005, China \\ ${ }^{2}$ Key Laboratory of Complex Systems and Intelligent Computing in Universities of Shandong (Linyi University), Linyi 276005, China \\ ${ }^{3}$ Department of Mathematics, Southeast University, Nanjing 210096, China \\ ${ }^{4}$ College of Science and Technology, North China Electric Power University, Baoding 071000, China \\ ${ }^{5}$ Department of Electrical and Computer Engineering, Faculty of Engineering, King Abdulaziz University, Jeddah 21589, Saudi Arabia
}

Correspondence should be addressed to Jianbao Zhang; jianbaozhang@163.com

Received 12 May 2018; Revised 27 June 2018; Accepted 25 July 2018; Published 9 August 2018

Academic Editor: Hiroaki Mukaidani

Copyright (C) 2018 Jianbao Zhang et al. This is an open access article distributed under the Creative Commons Attribution License, which permits unrestricted use, distribution, and reproduction in any medium, provided the original work is properly cited.

\begin{abstract}
Motivated by the quorum-sensing mechanism of bacteria, this paper modifies the network model by adding unknown parameters and noise disturbances and investigates the problem of outer synchronization via adaptive control. In case there exist three unknown parameters, updating laws are presented to identify the unknown parameters with help of Lyapunov stability theory, and the negative effects of noise disturbances are also compensated by designing adaptive controllers. In addition, we simplify the obtained conditions and carry out two succinct and utilitarian corollaries. Finally, numerical simulations are provided to show the validity of the obtained results.
\end{abstract}

\section{Introduction}

During the past decades, it has been discovered that bacteria, such as Escherichia coli, could communicate with each other through producing and monitoring one kind of signaling molecules $[1,2]$. The signaling molecules could diffuse into different bacteria or the environment, and the bacteria could coordinate their gene expression and activities in response to the concentration of the signaling molecules. Then, the bacteria are coupled with each other by the intercellular signaling molecules [3] and display various social behaviors such as behaving synchronously [4]. Obviously, the related researches have wide application prospects in biopharmaceutical industry and human health. Now, the mechanism of bacterial communication is widely known as quorum sensing, and more and more researchers began to study collective behavior caused by quorum sensing $[5,6]$. In this paper, we modify one of the previous network models coupled through quorum sensing and discuss a typical kind of collective behavior based on several recent methods developed in the fields of complex networks and nonlinear dynamics.

Recent years have witnessed the great development in the study of complex networks and its collective dynamics $[7,8]$. Synchronization is one of the most typical and most extensively studied kinds of collective dynamics, which implies the stability of zero solution of the synchronization error systems. Therefore, from the point of research methods, there are two main effective theoretical methods, i.e., the famous master stability function method $[9,10]$ and Lyapunov function method [11-13]. The former can be employed to discuss local stability of the synchronous state, and the latter can be used to explore global stability of the synchronous state. Up to now, dozens of different types of synchronization states have been proposed such as complete synchronization [14], cluster synchronization $[15,16]$, lag synchronization $[17,18]$, projection synchronization $[19,20]$, and outer synchronization [21-23]. Thereinto, outer synchronization has attracted many researchers' interest, which describes the synchronization 
between two or more networks. For instances, in a model of predator-prey interactions in ecological communities, all the predators form a network system and all the preys form another, and the two networks influence one another's evolution to keep the two species in check [24]. Recently, outer synchronization of the fractional order node dynamics was considered in [21], and outer synchronization under aperiodically adaptive intermittent control was considered in [22]. In many cases, networks can not realize a certain expected synchronization relying on just coupling interaction between different nodes [23]. Therefore, many different kinds of output control methods have been introduced, such as pinning control [25], sliding mode control [26, 27], adaptive control [28, 29], and state feedback control [30]. Thereinto, adaptive control could be used to design controllers for systems with uncertain parameters. Due to great demands from wide applications, many researches have been carried out to investigate synchronization induced by adaptive controllers [31]. It is worth pointing out that there are few researches focused on outer synchronization of networks coupled through quorum sensing.

Motivated by the above discussions, this paper investigates outer synchronization induced by adaptive controllers in quorum-sensing network. At first, we present a modified model of previous quorum-sensing network by adding noise disturbances in case there exist three unknown parameter vectors and the network topology is also unknown. Then, effective adaptive controllers are designed to realize outer synchronization, parameter estimations are designed to identify the unknown parameter vectors, and topology estimations are designed to identify unknown network topology. Based on Lyapunov function method and matrix theory, this paper proves that adaptive outer synchronization is achieved in the quorum-sensing network. To the best of our knowledge, there are few researches focused on this subject by a similar method. In our opinion, there is a certain degree of values both in theory and in practice.

The rest of this paper is organized as follows. In Section 2, the synthetic gene network model coupled through quorum sensing is introduced. In Section 3, several criteria are derived for outer synchronization including the construction of adaptive controllers and parameter estimations. In Section 4, some numerical examples are provided to illustrate the effectiveness of the obtained results. Finally, conclusions are given to summarize the contributions of the paper in Section 5 .

\section{Problem Formulation}

The synthetic gene network in Escherichia coli was first proposed by Garcia-Ojalvo J et al. [3]. Consider the network consisting of $N$ cells coupled through quorum sensing. Each cell consists of two basic parts illustrated in Figure 1 . The first part is composed of three genes $a, b, c$ that express their respective proteins $A, B, C$, which inhibit the transcription of the three genes $b, c, a$, in a cyclic way. The second part of each cell is another gene regulated by protein $A$, which produces a protein and synthesizes a small molecule known as an autoinducer $S_{i}$. The autoinducer $S_{i}$ can diffuse freely through the cell membrane, which activates the transcription of the genes in first part. For more detailed description, the reader is referred to previous articles $[32,33]$.

Now, we introduce the quorum-sensing network model. The dynamics of each node consists of the concentrations of three genes and their respective proteins, assume that the $i$ th cell is described by the following equations:

$$
\begin{aligned}
\dot{a}_{i}(t)= & -d_{1} a_{i}(t)+\beta_{6}\left[\mu_{6}+C_{i}^{m}(t)\right]^{-1}, \\
\dot{b}_{i}(t)= & -d_{2} b_{i}(t)+\beta_{4}\left[\mu_{4}+A_{i}^{m}(t)\right]^{-1}, \\
\dot{c}_{i}(t)= & -d_{3} c_{i}(t)+\beta_{5}\left[\mu_{5}+B_{i}^{m}(t)\right]^{-1} \\
& +\beta_{8} S_{i}(t)\left[\mu_{7}+S_{i}(t)\right]^{-1}, \\
\dot{A}_{i}(t)= & -d_{4} A_{i}(t)+\beta_{1} a_{i}(t), \\
\dot{B}_{i}(t)= & -d_{5} B_{i}(t)+\beta_{2} b_{i}(t), \\
\dot{C}_{i}(t)= & -d_{6} C_{i}(t)+\beta_{3} c_{i}(t),
\end{aligned}
$$

where $a_{i}(t), b_{i}(t)$, and $c_{i}(t)$ are the concentrations of $m R N A$ transcribed from genes $a, b, c$ in the $i$ th cell, respectively; $A_{i}(t), B_{i}(t)$, and $C_{i}(t)$ are the concentrations of the corresponding proteins, respectively; $S_{i}(t)$ and $S_{e}(t)$ are the concentrations of the autoinducer $A I$ inside the $i$ th cell and in the environment. The concentration dynamics of the autoinducer are governed by

$$
\begin{aligned}
& \dot{S}_{i}(t)=-d_{7} S_{i}(t)+\beta_{7} A_{i}(t)-\eta\left(S_{i}(t)-S_{e}(t)\right), \\
& \dot{S}_{e}(t)=-d_{e} S_{e}(t)+\frac{\eta_{e}}{N} \sum_{j=1}^{N}\left(S_{j}(t)-S_{e}(t)\right)
\end{aligned}
$$

where $i=1,2, \ldots, N$. In the multicell system (1)-(2), the parameters $d_{1}, d_{2}, \ldots, d_{7}$ and $d_{e}$ are the dimensionless degradation rates of the chemical molecules; $\beta_{1}, \beta_{2}, \beta_{3}$ are the translation rates of the proteins from the $m R N A s ; \beta_{4}, \beta_{5}, \beta_{6}$ are the dimensionless transcription rates in the absence of repressor; $\beta_{7}$ is the synthesis rate of $A I ; m=4$ is the Hill coefficient; $\beta_{8}$ is the maximal contribution to the gene $c$ transcription in the presence of saturating amounts of $A I ; \eta$ and $\eta_{e}$ measure the diffusion rate of $A I$ inward and outward the cell membrane. With the help of the quasi-steady state approximation $\dot{S}_{e}(t)=0$, one gets that the extracellular $A I$ concentration can be approximated as

$$
S_{e}(t)=\frac{q}{N} \sum_{j=1}^{N} S_{j}(t), \quad q=\frac{\eta_{e}}{d+\eta_{e}},
$$

which reduces (2) to the following form:

$$
\dot{S}_{i}(t)=-\left(d_{7}+\eta\right) S_{i}(t)+\beta_{7} A_{i}(t)+\frac{\eta q}{N} \sum_{j=1}^{N} S_{j}(t) .
$$

Equations (1)-(4) describe the concentration state of the $i$ th cell in the synthetic gene network model coupled through quorum sensing. 


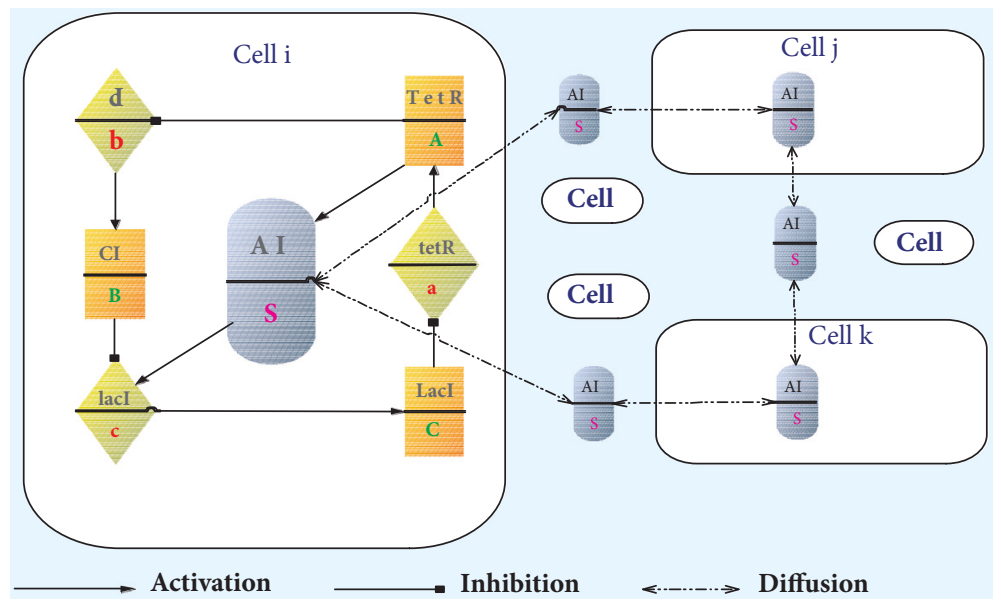

FIGURE 1: Scheme of the repressilator network coupled through signaling molecules, termed quorum sensing. The synchronization scheme of quorum sensing is based on the diffusion of autoinducers (AI) to and from the cells.

Motivated by the quorum-sensing network (1)-(4), we build up a network with three unknown parameter vectors and unknown network topology. The state dynamics are described by the following equations:

$$
\begin{aligned}
& \dot{x}_{i}(t)=f_{1}\left(x_{i}(t)\right) \alpha_{1}+f_{2}\left(x_{i}(t)\right) \alpha_{2}+\beta_{8} h\left(S_{i}(t)\right), \\
& \dot{S}_{i}(t)=-\alpha_{3} S_{i}(t)+\beta_{7} A_{i}(t)+\sum_{j=1}^{N} c_{i j} S_{j}(t),
\end{aligned}
$$

where $x_{i}(t)=\left(a_{i}(t), b_{i}(t), c_{i}(t), A_{i}(t), B_{i}(t), C_{i}(t)\right)^{\top}, \alpha_{1}=$ $\left(d_{1}, d_{2}, d_{3}, d_{4}, d_{5}, d_{6}\right)^{\top}, \alpha_{2}=\left(\beta_{6}, \beta_{4}, \beta_{5}, \beta_{1}, \beta_{2}, \beta_{3}\right)^{\top}$, and $\alpha_{3}=d_{7}+\eta$, and the diagonal matrix functions

$$
\begin{aligned}
& f_{1}\left(x_{i}(t)\right)=-\operatorname{diag}\left(a_{i}(t), b_{i}(t), c_{i}(t), A_{i}(t), B_{i}(t),\right. \\
& \left.C_{i}(t)\right), \\
& f_{2}\left(x_{i}(t)\right)=\operatorname{diag}\left(\left[\mu_{6}+C_{i}^{m}(t)\right]^{-1},\left[\mu_{4}+A_{i}^{m}(t)\right]^{-1},\right. \\
& \left.\quad\left[\mu_{5}+B_{i}^{m}(t)\right]^{-1}, a_{i}(t), b_{i}(t), c_{i}(t)\right), \\
& h\left(S_{i}(t)\right)=\left(0,0, S_{i}(t)\left[\mu_{7}+S_{i}(t)\right]^{-1}, 0,0,0\right)^{\top},
\end{aligned}
$$

where $i=1,2, \ldots, N$. To meet the demands of broad applications, the matrix $C=\left(c_{i j}\right)_{N \times N}$ is a coupling matrix denoting the network topology. The matrix element $c_{i j}$ is defined as follows: if there is a connection from node $i$ to node $j(i \neq j)$, then define the coupling strength as $c_{i j} \neq 0$; otherwise, $c_{i j}=0$. Let us assume that there are three unknown parameter vectors existing in the node dynamics, $\alpha_{1}, \alpha_{2}$, and $\alpha_{3}$, and the network topology matrix $C=\left(c_{i j}\right)_{N \times N}$ is also unknown.

In order to identify the unknown network topology and parameter vectors, we carry out another network model described by the following equations:

$$
\begin{aligned}
\dot{\bar{x}}_{i}(t)= & f_{1}\left(\bar{x}_{i}(t)\right) \bar{\alpha}_{1}(t)+f_{2}\left(\bar{x}_{i}(t)\right) \bar{\alpha}_{2}(t) \\
& +\beta_{8} h\left(\bar{S}_{i}(t)\right)+\Delta_{1}(t)+u_{1 i}(t), \\
\dot{\bar{S}}_{i}(t)= & -\bar{\alpha}_{3}(t) \bar{S}_{i}(t)+\beta_{7} \bar{A}_{i}(t)+\sum_{j=1}^{N} \bar{c}_{i j} \bar{S}_{j}+\Delta_{2}(t) \\
& +u_{2 i}(t),
\end{aligned}
$$

where $\bar{x}_{i}(t)=\left(\bar{a}_{i}(t), \bar{b}_{i}(t), \bar{c}_{i}(t), \bar{A}_{i}(t), \bar{B}_{i}(t), \bar{C}_{i}(t)\right)^{\top}, \bar{\alpha}_{1}(t)$, $\bar{\alpha}_{2}(t)$, and $\bar{\alpha}_{3}(t)$ are the estimations of the unknown parameter vectors $\alpha_{1}, \alpha_{2}$, and $\alpha_{3}$ in the network (5), $\Delta_{1}(t), \Delta_{2}(t)$ are the disturbances, and $u_{1 i}(t), u_{2 i}(t)$ are the controllers left to be designed later, $i=1,2, \ldots, N$.

\section{Adaptive Control Schemes for Outer Synchronization}

In this section, several criteria are derived for outer synchronization induced by adaptive control schemes. At first, we need to introduce the following two assumptions.

Assumption 1. For any $x=\left(x^{(1)}, x^{(2)}, \ldots, x^{(6)}\right)^{\top} \in R^{6}$ and $S \in R$, denote

$$
\begin{aligned}
& F\left(X, \alpha_{1}, \alpha_{2}, \alpha_{3}\right)=\left[\left(f_{1}(x) \alpha_{1}+f_{2}(x) \alpha_{2}+\beta_{8} h(S)\right)^{\top},\right. \\
& \left.-\alpha_{3} S+\beta_{7} x^{(4)}\right]^{\top} \in R^{7},
\end{aligned}
$$

where $X=\left(x^{\top}, S\right)^{\top} \in R^{7}$. There exists a positive constant $L$ such that the vector function $F\left(X, \alpha_{1}, \alpha_{2}, \alpha_{3}\right)$ satisfies that

$$
\begin{aligned}
& (Y-X)^{\top}\left[F\left(Y, \alpha_{1}, \alpha_{2}, \alpha_{3}\right)-F\left(X, \alpha_{1}, \alpha_{2}, \alpha_{3}\right)\right] \\
& \quad \leq L(Y-X)^{\top}(Y-X)
\end{aligned}
$$

for any $X, Y \in R^{7}$. 
Assumption 2. The disturbances $\Delta_{1}(t)$ and $\Delta_{2}(t)$ are bounded; i.e., there exist two positive constants $\rho_{1}, \rho_{2}$ such that

$$
\begin{aligned}
& \left\|\Delta_{1}(t)\right\| \leq \rho_{1}, \\
& \left\|\Delta_{2}(t)\right\| \leq \rho_{2} .
\end{aligned}
$$

Now, we design the state feedback controllers of the following form:

$$
\begin{aligned}
u_{1 i}(t)= & -\delta_{1 i}(t) e_{1 i}(t)-\gamma_{1 i}(t) \operatorname{sign}\left[e_{1 i}(t)\right], \\
\dot{\delta}_{1 i}(t)= & k_{1 i} e_{1 i}^{\top}(t) e_{1 i}(t), \quad k_{1 i}>0, \\
\dot{\gamma}_{1 i}(t)= & \xi_{1 i} e_{1 i}^{\top}(t) \operatorname{sign}\left[e_{1 i}(t)\right], \quad \xi_{1 i}>0, \\
u_{2 i}(t)= & -\delta_{2 i}(t) e_{2 i}(t)-\gamma_{2 i}(t) \operatorname{sign}\left[e_{2 i}(t)\right] \\
& +\sum_{j=1}^{N} p_{i j}(t) \bar{S}_{j}(t), \\
\dot{\delta}_{2 i}(t)= & k_{2 i} e_{2 i}^{2}(t), \quad k_{2 i}>0, \\
\dot{\gamma}_{2 i}(t)= & \xi_{2 i} \operatorname{sign}\left[e_{2 i}(t)\right] e_{2 i}(t), \quad \xi_{2 i}>0, \\
\dot{p}_{i j}(t)= & -\bar{S}_{j}(t) e_{2 i}(t),
\end{aligned}
$$

where $e_{1 i}(t)=\bar{x}_{i}(t)-x_{i}(t), e_{2 i}(t)=\bar{S}_{i}(t)-S_{i}(t)$, and $i=$ $1,2, \ldots, N$. Then, one can prove the following theorem based on Lyapunov function method and matrix theory.

Theorem 3. Suppose that Assumptions 1 and 2 hold, and the parameter estimations $\alpha_{1}(t), \alpha_{2}(t), \alpha_{3}(t)$ are designed as follows:

$$
\begin{aligned}
& \dot{\bar{\alpha}}_{1}(t)=-\sum_{j=1}^{N} f_{1}\left(\bar{x}_{j}(t)\right) e_{1 j}(t), \\
& \dot{\bar{\alpha}}_{2}(t)=-\sum_{j=1}^{N} f_{2}\left(\bar{x}_{j}(t)\right) e_{1 j}(t), \\
& \dot{\bar{\alpha}}_{3}(t)=\sum_{j=1}^{N} \bar{S}_{j}(t) e_{2 j}(t),
\end{aligned}
$$

and then the synthetic gene network (5)-(7) with controllers (11) and estimations (12) can achieve outer synchronization.

Proof. Denote $X_{i}(t)=\left(x_{i}^{\top}(t), S_{i}(t)\right)^{\top}, \bar{X}_{i}(t)=\left(\bar{x}_{i}^{\top}(t)\right.$, $\left.\bar{S}_{i}(t)\right)^{\top}, \Delta(t)=\left(\Delta_{1}^{\top}(t), \Delta_{2}(t)\right)^{\top}$, and $U_{i}(t)=\left(u_{1 i}^{\top}(t), u_{2 i}(t)\right)^{\top}$, and $\Gamma \in R^{7 \times 7}$ is the inner matrix implying the nodes are coupling through the 7th component, and then the synthetic gene network (5)-(7) can be rewritten as follows:

$$
\begin{aligned}
\dot{X}_{i}(t)= & F\left(X_{i}(t), \alpha_{1}, \alpha_{2}, \alpha_{3}\right)+\sum_{j=1}^{N} c_{i j} \Gamma X_{j}(t), \\
\dot{\bar{X}}_{i}(t)= & F\left(\bar{X}_{i}(t), \bar{\alpha}_{1}(t), \bar{\alpha}_{2}(t), \bar{\alpha}_{3}(t)\right) \\
& +\sum_{j=1}^{N} \bar{c}_{i j} \Gamma \bar{X}_{j}(t)+\Delta(t)+U_{i}(t),
\end{aligned}
$$

Let $E_{i}(t)=\bar{X}_{i}(t)-X_{i}(t)$, and the following error system can be obtained:

$$
\begin{aligned}
\dot{E}_{i}(t)= & F\left(\bar{X}_{i}(t), \bar{\alpha}_{1}(t), \bar{\alpha}_{2}(t), \bar{\alpha}_{3}(t)\right) \\
& -F\left(X_{i}(t), \alpha_{1}, \alpha_{2}, \alpha_{3}\right) \\
& +\sum_{j=1}^{N}\left[\bar{c}_{i j} \Gamma \bar{X}_{j}-c_{i j} \Gamma X_{j}\right]+\Delta(t)+U_{i}(t) .
\end{aligned}
$$

Using Assumption 1, one has

$$
\begin{aligned}
& E_{i}^{\top}(t)\left[F\left(\bar{X}_{i}(t), \bar{\alpha}_{1}(t), \bar{\alpha}_{2}(t), \bar{\alpha}_{3}(t)\right)\right. \\
& \left.-F\left(X_{i}(t), \alpha_{1}, \alpha_{2}, \alpha_{3}\right)\right] \leq E_{i}^{\top}(t) \\
& \cdot\left[F\left(\bar{X}_{i}(t), \bar{\alpha}_{1}(t), \bar{\alpha}_{2}(t), \bar{\alpha}_{3}(t)\right)\right. \\
& \left.-F\left(\bar{X}_{i}(t), \alpha_{1}, \alpha_{2}, \alpha_{3}\right)+L E_{i}(t)\right]=E_{i}^{\top}(t) \\
& \cdot\left[\left(\left(f_{1}\left(\bar{x}_{i}(t)\right) \widetilde{\alpha}_{1}(t)+f_{2}\left(\bar{x}_{i}(t)\right) \widetilde{\alpha}_{2}(t)\right)^{\top},\right.\right. \\
& \left.\left.-\widetilde{\alpha}_{3}(t) \bar{S}_{i}(t)\right)^{\top}+L E_{i}(t)\right]=\sum_{p=1}^{2} e_{1 i}^{\top}(t) f_{p}\left(\bar{x}_{i}(t)\right) \\
& \cdot \widetilde{\alpha}_{p}(t)-\widetilde{\alpha}_{3}(t) \bar{S}_{i}(t) e_{2 i}(t)+L \sum_{p=1}^{2} e_{p i}^{\top}(t) e_{p i}(t) .
\end{aligned}
$$

Consider the following Lyapunov function:

$$
\begin{aligned}
V(t) & =\frac{1}{2}\left\{\sum_{i=1}^{N} E_{i}^{\top}(t) E_{i}(t)+\sum_{p=1}^{3} \widetilde{\alpha}_{p}^{\top}(t) \widetilde{\alpha}_{p}(t)\right. \\
+ & \sum_{p=1}^{2} \sum_{i=1}^{N} \frac{1}{k_{p i}}\left[\delta_{p i}(t)-\delta_{p}^{*}\right]^{2} \\
+ & \sum_{p=1}^{2} \sum_{i=1}^{N} \frac{1}{\xi_{p i}}\left[\gamma_{p i}(t)-\gamma_{p}^{*}\right]^{2} \\
& \left.+\sum_{j=1}^{N} \sum_{i=1}^{N}\left[p_{i j}(t)+\bar{c}_{i j}-c_{i j}\right]^{2}\right\},
\end{aligned}
$$

where $\widetilde{\alpha}_{p}(t)=\bar{\alpha}_{p}(t)-\alpha_{p}, p=1,2,3$, where $\delta_{p}^{*}, \gamma_{p}^{*}, p=$ 1,2 , are positive constants chosen arbitrarily. With the help of controllers (11) and estimations (12), the derivative of $V(t)$ along the trajectories of (5)-(7) can be calculated as follows: 


$$
\begin{aligned}
\dot{V}(t)= & \sum_{i=1}^{N} E_{i}^{\top}(t) \dot{E}_{i}(t)-\sum_{p=1}^{2} \tilde{\alpha}_{p}^{\top}(t) \sum_{j=1}^{N} f_{p}\left(\bar{x}_{j}(t)\right) e_{1 j}(t) \\
& +\widetilde{\alpha}_{3}^{\top}(t) \sum_{j=1}^{N} \bar{S}_{j}(t) e_{2 j}(t) \\
& +\sum_{p=1}^{2} \sum_{i=1}^{N}\left[\delta_{p i}(t)-\delta_{p}^{*}\right] e_{p i}^{\top}(t) e_{p i}(t) \\
& +\sum_{p=1}^{2} \sum_{i=1}^{N}\left[\gamma_{p i}(t)-\gamma_{p}^{*}\right] e_{p i}^{\top}(t) \operatorname{sign}\left[e_{p i}(t)\right] \\
& -\sum_{j=1}^{N} \sum_{i=1}^{N}\left[p_{i j}(t)+\bar{c}_{i j}-c_{i j}\right] \bar{S}_{j}(t) e_{2 i}(t) .
\end{aligned}
$$

Noticing (14) and inequality (15), one has

$$
\begin{aligned}
\dot{V}(t) \leq & L \sum_{p=1}^{2} \sum_{i=1}^{N} e_{p i}^{\top}(t) e_{p i}(t) \\
& +\sum_{i=1}^{N} \sum_{j=1}^{N} e_{2 i}(t)\left[\bar{c}_{i j} \bar{S}_{j}(t)-c_{i j} S_{j}(t)\right] \\
& +\sum_{i=1}^{N} E_{i}^{\top}(t)\left[\Delta(t)+U_{i}(t)\right] \\
& +\sum_{p=1}^{2} \sum_{i=1}^{N}\left[\delta_{p i}(t)-\delta_{p}^{*}\right] e_{p i}^{\top}(t) e_{p i}(t) \\
& +\sum_{p=1}^{2} \sum_{i=1}^{N}\left[\gamma_{p i}(t)-\gamma_{p}^{*}\right] e_{p i}^{\top}(t) \operatorname{sign}\left[e_{p i}(t)\right] \\
& -\sum_{j=1}^{N} \sum_{i=1}^{N}\left[p_{i j}(t)+\bar{c}_{i j}-c_{i j}\right] e_{2 i}(t) \bar{S}_{j}(t) \\
\leq & L \sum_{p=1}^{2} \sum_{i=1}^{N} e_{p i}^{\top}(t) e_{p i}(t)+\sum_{i=1}^{N} \sum_{j=1}^{N} c_{i j} e_{2 i}(t) e_{2 j}(t) \\
& +\sum_{i=1}^{N} E_{i}^{\top}(t) \Delta(t)-\sum_{p=1}^{2} \sum_{i=1}^{N} \delta_{p}^{*} e_{p i}^{\top}(t) e_{p i}(t) \\
& \sum_{p=1}^{2} \sum_{i=1}^{N} \gamma_{p}^{*} e_{p i}^{\top}(t) s_{i g n}\left[e_{p i}(t)\right] \\
& \\
&
\end{aligned}
$$
gets

Denoting $e_{2}(t)=\left(e_{21}(t), e_{22}(t), \ldots, e_{2 N}(t)\right)^{\top} \in R^{N}$, one

$\dot{V}(t)$

$$
\begin{aligned}
\leq & \left(L-\delta_{1}^{*}\right) \sum_{i=1}^{N} e_{1 i}^{\top}(t) e_{1 i}(t) \\
& +e_{2}^{\top}(t)\left[\left(L-\delta_{2}^{*}\right) I_{N}+C\right] e_{2}(t) \\
& +\sum_{p=1}^{2} \sum_{i=1}^{N}\left[e_{p i}^{\top}(t) \Delta_{p}(t)-\gamma_{p}^{*} e_{p i}^{\top}(t) \operatorname{sign}\left[e_{p i}(t)\right]\right]
\end{aligned}
$$

where $\left\|e_{p i}(t)\right\|_{1}=e_{p i}^{\top}(t) \operatorname{sign}\left[e_{p i}(t)\right]$. Notice that the constants $\delta_{1}^{*}, \delta_{2}^{*}, \gamma_{1}^{*}, \gamma_{2}^{*}$ are chosen arbitrarily, and we can choose them sufficiently large such that $L-\delta_{1}^{*}<0, \rho_{p}-\gamma_{p}^{*}<0, p=$ 1,2 , and the matrix $\left(L-\delta_{2}^{*}\right) I_{N}+C$ is negative semidefinite. According to this, we have

$$
\dot{V}(t)<0 \text {. }
$$

Thus, based on Lyapunov stability methods, the error dynamical system (14) is globally asymptotically stable. Therefore, the synthetic gene network (5)-(7) with controllers (11) and estimations (12) achieves outer synchronization.

The proof is completed.

If one or two of the unknown parameter vectors in the network (5) are given constants, Theorem 3 still holds after modifying the conditions slightly. For instance, supposing that the parameter vectors $\alpha_{1}$ and $\alpha_{2}$ are given, we modify network (7) as follows:

$$
\begin{aligned}
\dot{\bar{x}}_{i}(t)= & f_{1}\left(\bar{x}_{i}(t)\right) \alpha_{1}+f_{2}\left(\bar{x}_{i}(t)\right) \alpha_{2}+\beta_{8} h\left(\bar{S}_{i}(t)\right) \\
& +\Delta_{1}(t)+u_{1 i}(t), \\
\dot{\bar{S}}_{i}(t)= & -\bar{\alpha}_{3}(t) \bar{S}_{i}(t)+\beta_{7} \bar{A}_{i}(t)+\sum_{j=1}^{N} \bar{c}_{i j} \bar{S}_{j}+\Delta_{2}(t) \\
& +u_{2 i}(t) .
\end{aligned}
$$

Then the following corollary holds.

Corollary 4. Suppose that Assumptions 1 and 2 hold. If the parameter estimation $\alpha_{3}(t)$ is designed as follows:

$$
\dot{\bar{\alpha}}_{3}(t)=\sum_{j=1}^{N} \bar{S}_{j}(t) e_{2 j}(t),
$$

then the synthetic gene network (5)-(21) with controllers (11) and estimations (22) can achieve outer synchronization.

If we do not consider the disturbances $\Delta_{1}(t)$ and $\Delta_{2}(t)$ in network (7), Theorem 3 still holds after modifying controllers (11) slightly. Then, we obtain the following corollary.

Corollary 5. Suppose that Assumptions 1 and 2 hold, and the disturbances in network (7) satisfy that $\Delta_{1}(t)=0$ and $\Delta_{2}(t)=$ 0 . If the controllers $u_{1 i}(t), u_{2 i}(t)$ are designed as follows: 


$$
\begin{aligned}
& u_{1 i}(t)=-\delta_{1 i}(t) e_{1 i}(t), \\
& \dot{\delta}_{1 i}(t)=k_{1 i} e_{1 i}^{\top}(t) e_{1 i}(t), \quad k_{1 i}>0, \\
& u_{2 i}(t)=-\delta_{2 i}(t) e_{2 i}(t)+\sum_{j=1}^{N} p_{i j}(t) \bar{S}_{j}(t), \\
& \dot{\delta}_{2 i}(t)=k_{2 i} e_{2 i}^{2}(t), \quad k_{2 i}>0, \\
& \dot{p}_{i j}(t)=-\bar{S}_{j}(t) e_{2 i}(t),
\end{aligned}
$$

where $i=1,2, \ldots, N$, then the synthetic gene network (5)(7) with controllers (23) and estimations (12) can achieve outer synchronization.

The proof of Corollaries 4 and 5 is similar to that of Theorem 3; therefore, it is omitted here.

\section{Numerical Simulations}

In this section, we carry out some numerical simulations on the following synthetic gene network consisting of 6 cells:

$$
\begin{aligned}
\dot{x}_{i}(t)= & f_{1}\left(x_{i}(t)\right) \alpha_{1}+f_{2}\left(x_{i}(t)\right) \alpha_{2}+\beta_{8} h\left(S_{i}(t)\right), \\
\dot{\bar{x}}_{i}(t)= & f_{1}\left(\bar{x}_{i}(t)\right) \bar{\alpha}_{1}(t)+f_{2}\left(\bar{x}_{i}(t)\right) \bar{\alpha}_{2}(t) \\
& +\beta_{8} h\left(\bar{S}_{i}(t)\right)+\Delta_{1}(t)+u_{1 i}(t), \\
\dot{S}_{i}(t)= & -\alpha_{3} S_{i}(t)+\beta_{7} A_{i}(t)+\sum_{j=1}^{6} c_{i j} S_{j}(t), \\
\dot{\bar{S}}_{i}(t)= & -\bar{\alpha}_{3}(t) \bar{S}_{i}(t)+\beta_{7} \bar{A}_{i}(t)+\sum_{j=1}^{6} \bar{c}_{i j} \bar{S}_{j}+\Delta_{2}(t) \\
& +u_{2 i}(t),
\end{aligned}
$$

where the functions $f_{1}\left(x_{i}(t)\right), f_{2}\left(x_{i}(t)\right), h\left(S_{i}(t)\right)$ are defined in network (5), the parameters are given as $\left(\mu_{4}, \mu_{5}, \mu_{6}, \mu_{7}\right)=$ $(0.2,0.2,0.2,0.2), m=4, \beta_{7}=0.018, \beta_{8}=1, \Delta_{1}(t), \Delta_{2}(t)$ are the disturbances, and the coupling matrices are given as

$$
\begin{aligned}
& C=\left(\begin{array}{cccccc}
-5 & 1 & 2 & 0 & 1 & 1 \\
1 & -4 & 0 & 1 & 2 & 0 \\
2 & 0 & -6 & 2 & 0 & 2 \\
0 & 1 & 2 & -4 & 1 & 0 \\
1 & 2 & 0 & 1 & -4 & 0 \\
1 & 0 & 2 & 0 & 0 & -3
\end{array}\right), \\
& \bar{C}=\left(\begin{array}{cccccc}
-6 & 2 & 0 & 1 & 1 & 2 \\
2 & -6 & 1 & 2 & 1 & 0 \\
0 & 1 & -5 & 1 & 2 & 1 \\
1 & 2 & 1 & -5 & 0 & 1 \\
1 & 1 & 2 & 0 & -5 & 1 \\
2 & 0 & 1 & 1 & 1 & -5
\end{array}\right) .
\end{aligned}
$$

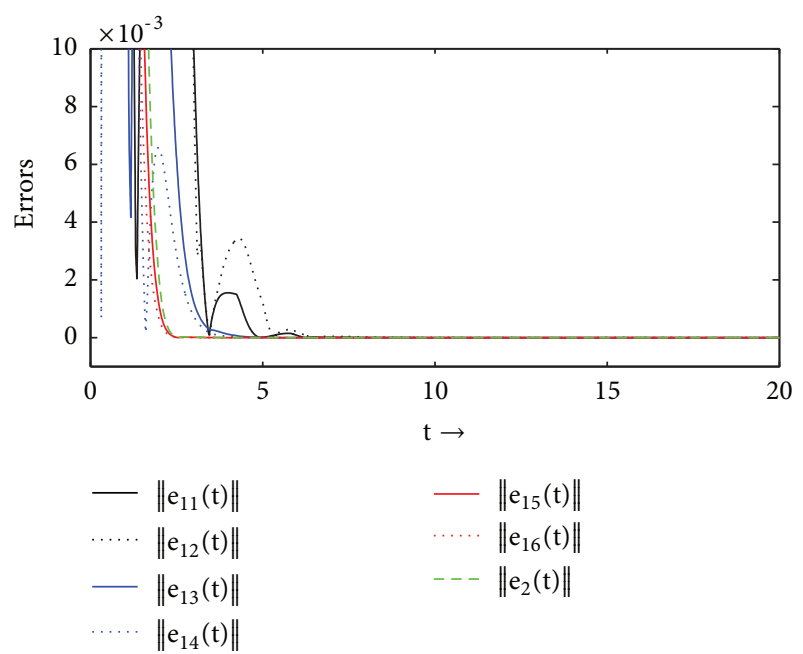

Figure 2: Time evolutions of synchronization errors $\left\|e_{1 i}(t)\right\|$ and $\left\|e_{2}(t)\right\|, i=1,2, \ldots, 6$.

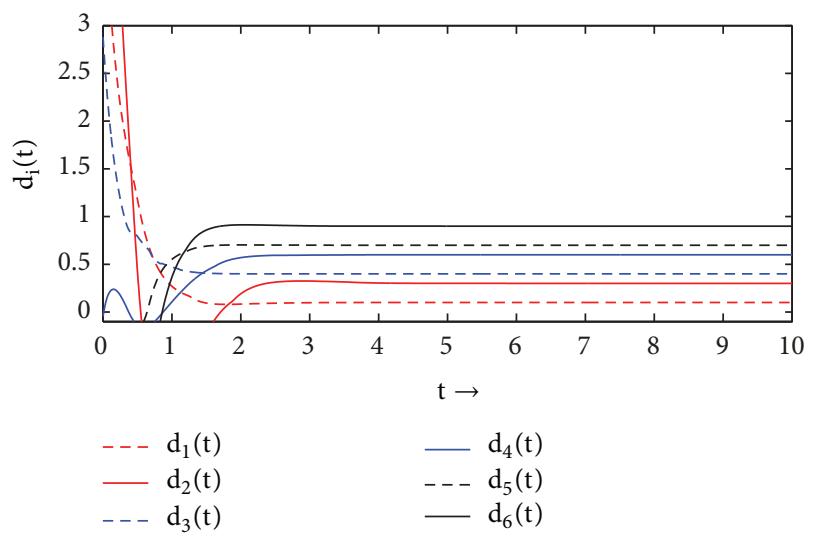

FIgURE 3: Time evolutions of the parameter estimations $\bar{\alpha}_{1}(t)=$ $\left(d_{1}(t), d_{2}(t), \ldots, d_{6}(t)\right)^{\top}$ in $(12)$.

Consider the actual meaning of the network, the true values of the unknown parameters are

$$
\begin{aligned}
& \alpha_{1}=(0.1,0.3,0.4,0.6,0.7,0.9)^{\top}, \\
& \alpha_{2}=(2,1.9,1.5,0.2,0.6,0.9)^{\top}, \\
& \alpha_{3}=0.42,
\end{aligned}
$$

estimations (12) are adopted for $\bar{\alpha}_{1}(t), \bar{\alpha}_{2}(t), \bar{\alpha}_{3}(t)$, and controllers (11) are adopted for $u_{1 i}(t), u_{2 i}(t)$.

By setting the initial values of network (24) randomly in $[0,1]$ and with the feedback gain taken as $k_{1 i}=k_{2 i}=$ 1 , we plot Figure 2 to show the time evolutions of outer synchronization errors $\left\|e_{1 i}(t)\right\|=\left\|\bar{x}_{i}(t)-x_{i}(t)\right\|$ and $\left\|e_{2 i}(t)\right\|=$ $\left\|\bar{S}_{i}(t)-S_{i}(t)\right\|, i=1,2, \ldots, 6$. It can be seen that the two errors both go to zero quickly after a short transient period, and network (24) reaches outer synchronization. Figure 3 depicts the time evolutions of the parameter estimations $\bar{\alpha}_{1}(t)$, which displays the perfect identification performance. Figure 4 shows the time evolutions of the parameter estimations 


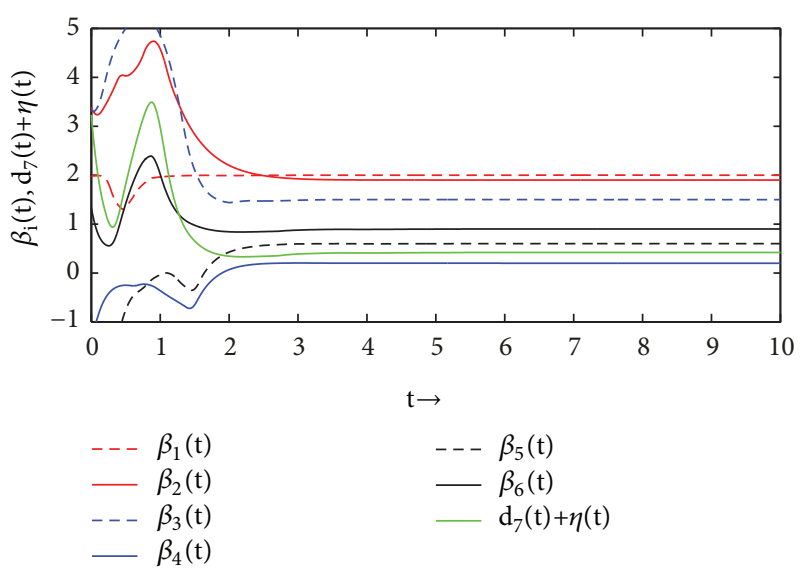

FIGURE 4: Time evolutions of the parameter estimations $\bar{\alpha}_{2}(t)=$ $\left(\beta_{1}(t), \beta_{2}(t), \ldots, \beta_{6}(t)\right)^{\top}$ and $\bar{\alpha}_{3}(t)=d_{7}(t)+\eta(t)$ in (12).

$\bar{\alpha}_{2}(t), \bar{\alpha}_{3}(t)$, and further illustrates the effectiveness of the parameter estimations (12). From the three figures, it is clearly observed that the unknown parameters of the network is estimated successfully by the control schemes of Theorem 3.

\section{Conclusions}

This paper builds a model of quorum-sensing network with disturbances, unknown parameter vector, and network topology and investigates the problem of outer synchronization between two quorum-sensing networks. In case that some systems' parameters are unknown in actual applications, adaptive parameter updating laws are designed to estimate the true values of those unknown parameters. Similarly, updating laws are also presented for the unknown elements of the network coupling matrix. Finally, some adaptive controllers are adopted to realize outer synchronization between two quorum-sensing networks. The validity of the proposed control schemes and updating laws is demonstrated by several numerical simulations.

\section{Data Availability}

The authors affirm that all data necessary for confirming the conclusions of the article are present within the article.

\section{Conflicts of Interest}

The authors declare that there are no conflicts of interest regarding the publication of this article.

\section{Acknowledgments}

Project is supported by National Natural Science Foundation of China (nos. 11447005, 61771230, and 61663006), Shandong Provincial Key Research and Development Program of China (no. 2017CXGC0701), Shandong Provincial Natural Science Foundation (no. ZR2016FM40, ZR2016JL021), Jiangsu Planned Projects for Postdoctoral Research Funds (no. 1701017A), and the Fundamental Research Funds for the Central Universities (no. 9161718004).

\section{References}

[1] J. D. Dockery and J. P. Keener, "A mathematical model for quorum sensing in Pseudomonas aeruginosa," Bulletin of Mathematical Biology, vol. 63, no. 1, pp. 95-116, 2001.

[2] M. E. Taga and B. L. Bassler, "Chemical communication among bacteria," Proceedings of the National Acadamy of Sciences of the United States of America, vol. 100, no. 24, pp. 14549-14554, 2003.

[3] J. Garcia-Ojalvo, M. B. Elowitz, and S. H. Strogatz, "Modeling a synthetic multicellular clock: repressilators coupled by quorum sensing," Proceedings of the National Acadamy of Sciences of the United States of America, vol. 101, no. 30, pp. 10955-10960, 2004.

[4] R. Wang and L. Chen, "Synchronizing genetic oscillators by signaling molecules," Journal of Biological Rhythms, vol. 20, no. 3, pp. 257-269, 2005.

[5] E. V. Nikolaev and E. D. Sontag, "Quorum-sensing synchronization of synthetic toggle switches: a design based on monotone dynamical systems theory," PLoS Computational Biology, vol. 12, no. 4, Article ID e1004881, 2016.

[6] B. L. Bassler, "Quorum sensing and its control," The FASEB Journal, vol. 30, no. 1, 2016.

[7] W. Sun, S. Wang, G. Wang, and Y. Wu, "Lag synchronization via pinning control between two coupled networks," Nonlinear Dynamics, vol. 79, no. 4, pp. 2659-2666, 2015.

[8] P. Zhou, S. Cai, J. Shen, and Z. Liu, "Adaptive exponential cluster synchronization in colored community networks via aperiodically intermittent pinning control," Nonlinear Dynamics, vol. 92, no. 3, pp. 905-921, 2018.

[9] L. M. Pecora and T. L. Carroll, "Master stability functions for synchronized coupled systems," Physical Review Letters, vol. 80, no. 10, pp. 2109-2112, 1998.

[10] A. Brechtel, P. Gramlich, D. Ritterskamp, B. Drossel, and T. Gross, "Master stability functions reveal diffusion-driven pattern formation in networks," Physical Review E: Statistical, Nonlinear, and Soft Matter Physics, vol. 97, no. 3, Article ID 032307, 2018.

[11] Z.-Y. Sun, M.-M. Yun, and T. Li, "A new approach to fast global finite-time stabilization of high-order nonlinear system," Automatica, vol. 81, pp. 455-463, 2017.

[12] X.-J. Xie, Z.-J. Li, and K. Zhang, "Semi-global output feedback control for nonlinear systems with uncertain time-delay and output function," International Journal of Robust and Nonlinear Control, vol. 27, no. 15, pp. 2549-2566, 2017.

[13] Y. Li, Y. Sun, and F. Meng, "New criteria for exponential stability of switched time-varying systems with delays and nonlinear disturbances," Nonlinear Analysis: Hybrid Systems, vol. 26, pp. 284-291, 2017.

[14] L. M. Pecora and T. L. Carroll, "Synchronization in chaotic systems," Physical Review Letters, vol. 64, no. 8, pp. 821-824, 1990.

[15] A. Hu, J. Cao, M. Hu, and L. Guo, "Cluster synchronization in directed networks of non-identical systems with noises via random pinning control," Physica A: Statistical Mechanics and its Applications, vol. 395, pp. 537-548, 2014.

[16] J. Zhang, Z. Ma, and G. Chen, "Robustness of cluster synchronous patterns in small-world networks with inter-cluster co-competition balance," Chaos: An Interdisciplinary Journal of Nonlinear Science, vol. 24, no. 2, Article ID 023111, 2014. 
[17] H. Liu, W. Sun, and G. Al-mahbashi, "Parameter identification based on lag synchronization via hybrid feedback control in uncertain drive-response dynamical networks," Advances in Difference Equations, vol. 2017, no. 1, article 122, 2017.

[18] Z. Wang, L. Huang, and X. Yang, "Adaptive modified function projective lag synchronization for two different chaotic systems with stochastic unknown parameters," Mediterranean Journal of Mathematics, vol. 13, no. 3, pp. 1391-1405, 2016.

[19] Abdesselem Boulkroune, Sarah Hamel, Farouk Zouari, Abdelkrim Boukabou, and Asier Ibeas, "Output-feedback controller based projective lag-synchronization of uncertain chaotic systems in the presence of input nonlinearities," Mathematical Problems in Engineering, vol. 2017, Article ID 8045803, 12 pages, 2017.

[20] J. Guan, "Function projective synchronization of a class of chaotic systems with uncertain parameters," Mathematical Problems in Engineering, vol. 2012, Article ID 431752, 5 pages, 2012.

[21] M. M. Asheghan and J. n. Míguez, "Robust global synchronization of two complex dynamical networks," Chaos: An Interdisciplinary Journal of Nonlinear Science, vol. 23, no. 2, Article ID 023108, 2013.

[22] S. Cai, X. Lei, and Z. Liu, "Outer synchronization between two hybrid-coupled delayed dynamical networks via aperiodically adaptive intermittent pinning control," Complexity, vol. 21, no. S2, pp. 593-605, 2016.

[23] L. Wang, J. Zhang, and W. Sun, "Adaptive outer synchronization and topology identification between two complex dynamical networks with time-varying delay and disturbance," IMA Journal of Mathematical Control and Information, 2018.

[24] X. Wu, W. X. Zheng, and J. Zhou, "Generalized outer synchronization between complex dynamical networks," Chaos: An Interdisciplinary Journal of Nonlinear Science, vol. 19, no. 1, Article ID 013109, 9 pages, 2009.

[25] C. Yu, J. Qin, and H. Gao, "Cluster synchronization in directed networks of partial-state coupled linear systems under pinning control," Automatica, vol. 50, no. 9, pp. 2341-2349, 2014.

[26] X. Chen, J. H. Park, J. Cao, and J. Qiu, "Adaptive synchronization of multiple uncertain coupled chaotic systems via sliding mode control," Neurocomputing, vol. 273, pp. 9-21, 2018.

[27] Junbiao Guan and Kaihua Wang, "Sliding mode control and modified generalized projective synchronization of a new fractional-order chaotic system," Mathematical Problems in Engineering, vol. 2015, Article ID 941654, 9 pages, 2015.

[28] W. Sun and L. Peng, "Observer-based robust adaptive control for uncertain stochastic Hamiltonian systems with state and input delays," Lithuanian Association of Nonlinear Analysts. Nonlinear Analysis: Modelling and Control, vol. 19, no. 4, pp. 626-645, 2014

[29] Z.-Y. Sun, C.-H. Zhang, and Z. Wang, "Adaptive disturbance attenuation for generalized high-order uncertain nonlinear systems," Automatica, vol. 80, pp. 102-109, 2017.

[30] X. Xie and M. Jiang, "Output feedback stabilization of stochastic feedforward nonlinear time-delay systems with unknown output function," International Journal of Robust and Nonlinear Control, vol. 28, no. 1, pp. 266-280, 2018.

[31] Yuling Li, Yixin Yin, and Sen Zhang, "Adaptive Control of Delayed Teleoperation Systems with Parameter Convergence," Mathematical Problems in Engineering, vol. 2018, Article ID 1046419, 7 pages, 2018.
[32] M. B. Elowitz and S. Leibier, "A synthetic oscillatory network of transcriptional regulators," Nature, vol. 403, no. 6767, pp. 335338, 2000.

[33] J. Zamora-Munt, C. Masoller, J. Garcia-Ojalvo, and R. Roy, "Crowd synchrony and quorum sensing in delay-coupled lasers," Physical Review Letters, vol. 105, no. 26, Article ID 264101, 2010. 


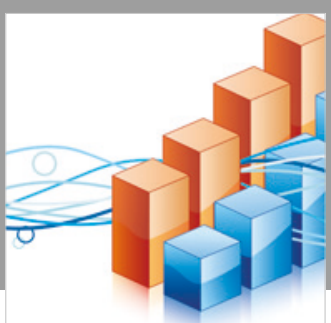

Advances in

Operations Research

\section{-n-m}
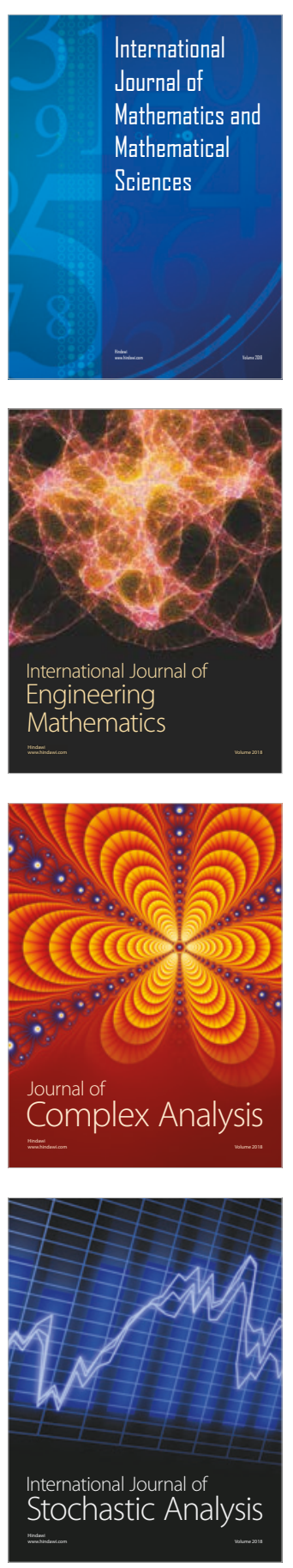
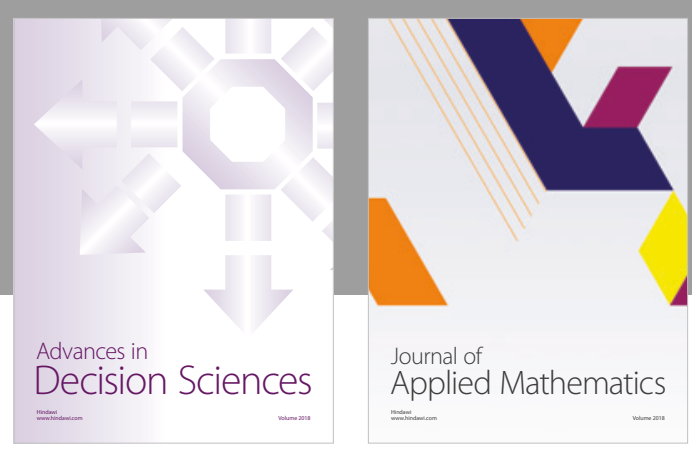

Journal of

Applied Mathematics
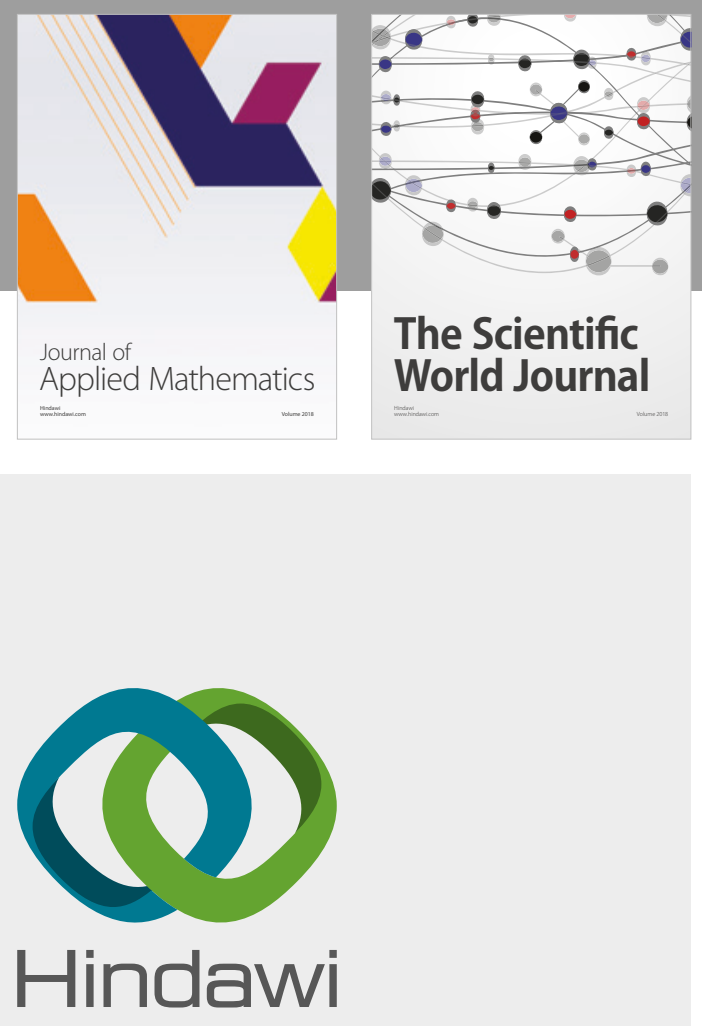

Submit your manuscripts at

www.hindawi.com

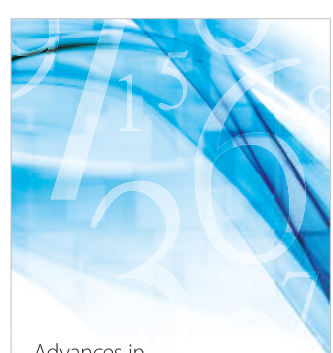

Advances in
Numerical Analysis
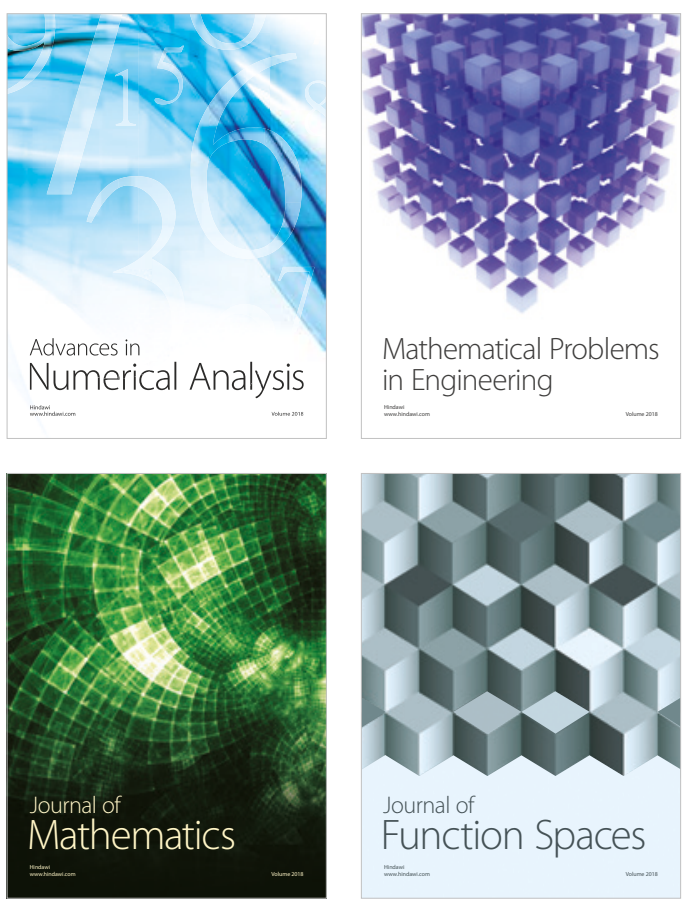

Mathematical Problems in Engineering

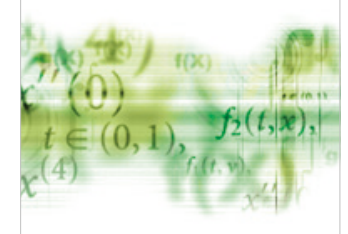

International Journal of

Differential Equations

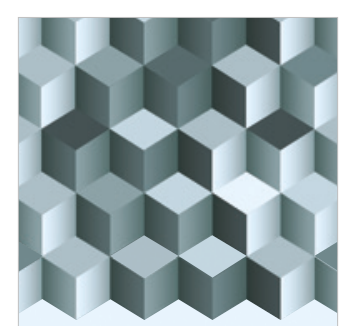

Journal of

Function Spaces

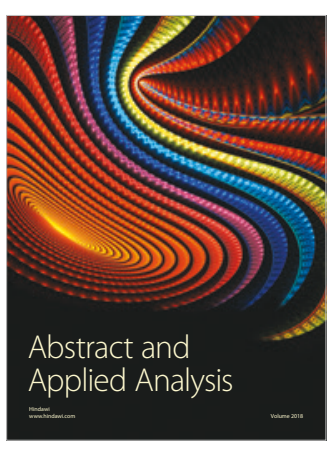

The Scientific

World Journal

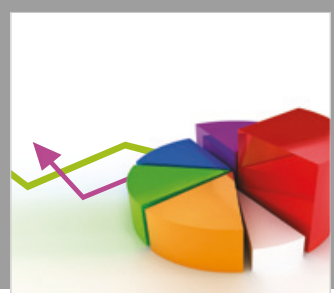

Journal of

Probability and Statistics
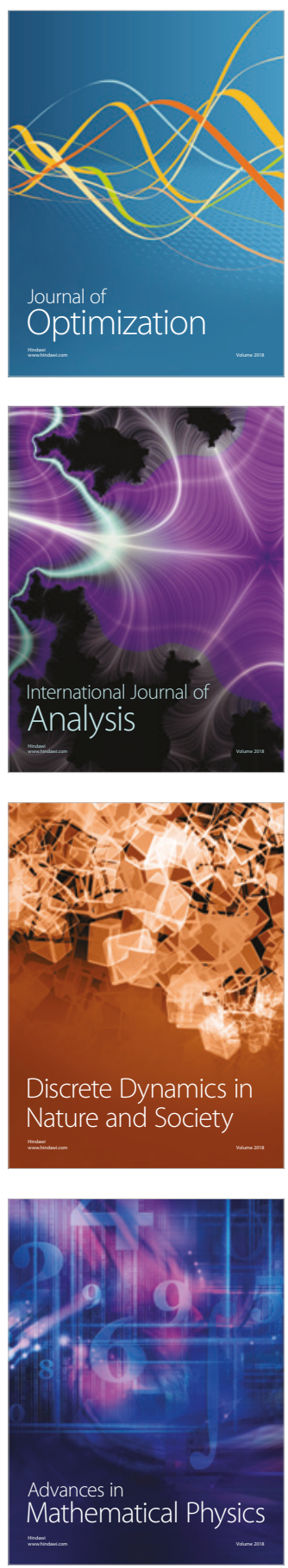